\title{
Inflation with Low Reheat Temperature and Cosmological Constraint on Stable Charged Massive Particles
}

\author{
Atsushi Kudo and Masahiro Yamaguchi* \\ Department of Physics, Tohoku University, Sendai 980-8578, Japan
}

(March 2001)

\begin{abstract}
Abundance of stable charged massive particles (CHAMPs) is severely constrained by their searches inside sea water. We examine whether inflation with low reheat temperature can sufficiently dilute the abundance to evade this severe bound on CHAMPs, by taking into account their production and annihilation in a plasma during reheating phase. We argue that the abundance of the CHAMPs with mass less than $1 \mathrm{TeV}$ will exceed the experimental bound, ruling out these CHAMPs, especially the possibility of the superpartner of tau lepton as the stable lightest superparticle. On the other hand, it is found that the CHAMP much heavier than $1 \mathrm{TeV}$ can survive the bound, if the reheat temperature is low enough.
\end{abstract}

*e-mail: yama@tuhep.phys.tohoku.ac.jp 
Extensions of standard model of particle physics often have stable charged massive particles (CHAMPs) [1,2]. For instance in the minimal supersymmetric standard model (MSSM) [3], the lightest superparticle (LSP) becomes absolutely stable if the R-parity is conserved. In some scenarios of supersymmetry breaking, the superpartner of tau lepton that is called stau can be the LSP. Another example is the lightest messenger field in the gauge mediated supersymmetry breaking, which may be charged and stable in some regions of the parameter space [四].

In the usual thermal history of the Universe where the radiation dominated era started at a very high temperature, CHAMPs interacted among themselves and with other particles very rapidly and thus they were in the thermal equilibrium. As the temperature went down, they got non-relativistic and then they were frozen out when the annihilation rate among them became smaller than the expansion rate of the Universe. Their relic abundance can be computed in a standard matter [5], which implies that they would constitute a significant portion of the total mass of the Universe. Such abundant CHAMPs must be ruled out, as the CHAMPs density is severely constrained by the null results of CHAMPs searches inside sea water [6 10]. Therefore models which predict existence of such stable charged massive particles are normally thought to be excluded.

The abundance of CHAMPs may be drastically diluted when the reheat temperature of the Universe is very low. Here the reheat temperature is the temperature when radiation dominated era commences after inflationary epoch and subsequent decays of inflaton during its damped oscillation. The issue of the relic abundance of stable massive particles in this setting was recently discussed by Ref. [11]. (See also [12].) It was shown that the relic abundance can be substantially reduced compared to the case of standard thermal history. The reason of the reduction is two-folds. Stable particles are produced in a plasma during reheating process, [7 where relativistic particles are supplied by decays of inflaton. If their production cross section is not large enough, then they do not reach their thermal equilibrium and thus the abundance becomes small. Even if the production is effective, the freeze-out (of the annihilation) can take place before the reheating process ends. If so, the relic abundance, i.e. the mass density relative to entropy density, is diluted by subsequent entropy production during the reheating.

In this paper, we apply the argument of Ref. [11] to the case of CHAMPs and examine whether the CHAMPs are capable of surviving the very strong constraints coming from the searches for them. We will show that, with reasonable assumptions on the distribution of the CHAMPs inside our galaxy, the CHAMPs lighter than about $1 \mathrm{TeV}$ should be excluded. Thus the stable stau LSP which is expected to have an electroweak scale mass is excluded even with this unconventional thermal history of the Universe. On the contrary, we find that the cosmological constraint can be evaded if the CHAMP's mass is much heavier than $1 \mathrm{TeV}$. The latter result implies that the stable lightest messenger particle in the gauge mediated supersymmetry breaking scenario is not necessarily a cosmological embarrassment if the reheat temperature is sufficiently low.

As inflationary expansion ends, an inflaton field starts its damped coherent oscillation

\footnotetext{
1 The maximum temperature of the plasma is much higher than the reheat temperature itself and thus heavy particles can be produced.
} 
which dominates the energy density of the Universe.2 The energy stored in the form of the inflaton oscillation is released to the radiation (i.e. relativistic particles) by inflaton decays. The CHAMPs we are considering here are pair produced the plasma of the radiation. Here we assume that the inflaton decays do not contain the CHAMPs at all and they are solely produced in the thermal bath. The set of Boltzmann equations and Friedmann equation relevant to our calculation are [1]

$$
\begin{aligned}
\frac{d \rho_{\phi}}{d t} & =-3 H \rho_{\phi}-\Gamma_{\phi} \rho_{\phi} \\
\frac{d \rho_{R}}{d t} & =-4 H \rho_{R}+\Gamma_{\phi} \rho_{\phi}+2\langle\sigma v\rangle\left\langle E_{X}\right\rangle\left[n_{X}^{2}-\left(n_{X}^{e q}\right)^{2}\right] \\
\frac{d n_{X}}{d t} & =-3 H n_{X}-\langle\sigma v\rangle\left[n_{X}^{2}-\left(n_{X}^{e q}\right)^{2}\right] \\
H^{2} & \equiv\left(\frac{\dot{a}}{a}\right)^{2}=\frac{8 \pi}{3 m_{P l}^{2}}\left(\rho_{\phi}+\rho_{R}+\rho_{X}\right)
\end{aligned}
$$

where $\phi$ is an inflaton field, $\rho_{\phi}$ and $\Gamma_{\phi}$ are its energy density and decay width respectively, $X$ is a CHAMP and $n_{X}$ is its number density, $n_{X}^{e q}$ that of equilibrium, $\left\langle E_{X}\right\rangle$ the thermal-average of CHAMP's energy, $\rho_{R}$ is an energy density of radiation, $H$ is an expansion parameter of the Universe, $a$ is a scale factor, $m_{P l}$ is a Planck scale, and $\langle\sigma v\rangle$ is a thermal-averaged annihilation cross section times their relative velocity. The second term of the r.h.s. of Eq. (3) represents annihilation and production of $X$ particles in the plasma of relativistic particles created by inflaton decays. In this paper we have assumed that the inflaton field obeys simple exponential decays, and also that the relativistic particles produced by the inflaton decays immediately reach their thermal equilibrium distributions. To check the validity of these assumptions is a very interesting issue, but is beyond the scope of the present paper.

The annihilation cross section of the CHAMPs would be model-dependent. For instance that of the stau LSP in the MSSM has been discussed in Ref. [14] in a different context. The dominant contributions come from the annihilation processes into $\gamma \gamma$ and $Z \gamma$. To be specific, we use

$$
\langle\sigma v\rangle=\frac{4 \pi \alpha^{2}}{m_{X}^{2}}+\mathcal{O}\left(T / m_{X}\right),
$$

with $\alpha$ the fine structure constant and $m_{X}$ the mass of the $X$ particle.

The relic abundance of the CHAMPs is computed by solving the set of Eqs (1-4) numerically under the following initial conditions:

$$
\rho_{\phi}\left(t_{i}\right)=\rho_{\phi i} \quad ; \quad \rho_{R}\left(t_{i}\right)=0 ; n_{X}\left(t_{i}\right)=0 ; a\left(t_{i}\right)=a_{i},
$$

where $t_{i}$ is the time when the damped oscillation of the inflaton field starts just after the end of inflationary epoch, and $\rho_{\phi i}$ is the energy density of the inflaton at that time. We take $\rho_{\phi i}$ sufficiently large so that the final answer will not depend on this initial value.

\footnotetext{
2 The inflation we are considering is not necessarily a primordial inflation, but can be a subsequent mini-inflation such as thermal inflation [13].
} 
To present our results, it is convenient to define the reheat temperature as

$$
T_{R} \equiv\left(\frac{45}{4 \pi^{3}} \frac{1}{g_{*}\left(T_{R}\right)}\right)^{1 / 4} \sqrt{m_{P l} \Gamma_{\phi}},
$$

where $g_{*}(T)$ is the effectively relativistic degree of freedom at temperature $T$.

In fig. 1 we show the relic abundance of the CHAMPs $X$ produced during the reheating procedure. We plot the constant contours of $\Omega_{X} h^{2}$, where $\Omega_{X}$ is the ratio of the mass density of the $X$ particle relative to the critical density of the Universe, in the $m_{X}-T_{R}$ plane. Note that the abundance we compute here is an average over the whole Universe. When the mass much exceeds $1 \mathrm{TeV}$, the production (annihilation) cross section is small so that the $X$ particles do not reach the equilibrium. On the other hand, the CHAMP with weak scale mass has reasonable cross cross section to establish the equilibrium. In this case the entropy production associated to the reheating of the Universe dilutes the relic abundance. We find as the reheating temperature goes down the relic abundance becomes drastically small, as we anticipated.

The abundance of the CHAMPs is severely constrained by null results of CHAMPs searches in ocean water. Various experiments were done, giving severe bounds on the contamination of CHAMPs [6 [10]. They are summarized in Table 1, which we quote from [15]. Typically for masses $m_{X} \simeq 100-1000 \mathrm{GeV}$, the number density of the CHAMPs with electric charge +1 relative to that of hydrogen atoms inside the sea water is tightly constrained

$$
\left(\frac{n_{X}}{n_{H}}\right)_{\text {Earth }} \lesssim 10^{-28}-10^{-29},
$$

while for heavier CHAMPs, the bound becomes weaker:

$$
\left(\frac{n_{X}}{n_{H}}\right)_{\text {Earth }} \lesssim 10^{-14} .
$$

Light charged particles can lose their energy by bremsstrahlung. For heavy CHAMPs, however, it is not effective. More important is Coulomb scattering with protons. According to Ref. [1] the relaxation time of CHAMPs through Coulomb scatterings with protons is estimated as $\tau \sim 5 \times 10^{8}\left(m_{X} / 10 \mathrm{TeV}\right)$ yr. Since the dynamical time scale for our galaxy formation is about $10^{9}$ yrs, CHAMPs with mass $m_{X} \lesssim 20 \mathrm{TeV}$ will lose their energy and fall into the galactic disc. Heavier CHAMPs will remain in the halo of the galaxy.

Let us now estimate how many CHAMPs we can expect inside the sea water. When the CHAMPs are in the halo $\left(m_{X} \gtrsim 20 \mathrm{TeV}\right)$, the flux of the positively charged CHAMPs? is given as

$$
\phi_{+}=\frac{\rho_{\text {halo }}}{m_{X}} v_{\text {halo }} f_{+}
$$

\footnotetext{
${ }^{3}$ Here the positive CHAMPs consist of $X^{+}$and possibly $X^{-}$bound with a $\alpha$ nucleus. To obtain a conservative bound on the relic abundance, we will ignore the latter contribution.
} 
where $\rho_{\text {halo }} \simeq 0.3 \mathrm{GeV} / \mathrm{cm}^{3}$ is the energy density of the galactic halo nearby the Earth, and $v_{\text {halo }} \simeq 300 \mathrm{~km} / \mathrm{sec}$ is a characteristic velocity of the halo relative to the Earth (See for example [16]). And $f_{+}$is the ratio of the mass density of the positive CHAMPs to that of the dark matter in the halo. These positive CHAMPs lose energy by collisions with atmosphere of the Earth and they are accumulated in the ocean water. The number density of the positive CHAMPs in the sea water is

$$
n_{X}=\frac{\phi_{+} t_{a c c}}{4 d}
$$

where $d \simeq 2.6 \mathrm{~km}$ is the average ocean depth over all of the Earth's surface and $t_{a c c}$ is the accumulation time of the CHAMPs inside the sea water, which we will assume to be the age of the ocean $t_{a c c} \simeq 3 \times 10^{9}$ yrs. It follows from Eqs. (10) and (11) that

$$
\left(\frac{n_{X}}{n_{H}}\right)_{\text {Earth }} \simeq 4 \times 10^{-15}\left(\frac{\mathrm{GeV}}{m_{X}}\right)\left(\frac{t_{a c c}}{\mathrm{yr}}\right) f_{+} .
$$

To evaluate the fraction $f_{+}$, we assume that the local fraction of the CHAMP's energy density relative to that of the (cold) dark matter in the halo nearby the Earth traces its global fraction in the whole Universe. Thus

$$
f_{+} \simeq \frac{1}{2} \frac{\Omega_{X} h^{2}}{\Omega_{D M} h^{2}}
$$

In the following we take the mass density of the dark matter $\Omega_{D M} \simeq 0.35$ and $h \simeq 0.7$.

Combining all, we obtain for $m_{X} \gtrsim 20 \mathrm{TeV}$

$$
\left(\frac{n_{X}}{n_{H}}\right)_{\text {Earth }} \simeq 3 \times 10^{-5}\left(\frac{\mathrm{GeV}}{m_{X}}\right) \Omega_{X} h^{2} .
$$

When the CHAMPs are in the galactic disc $\left(m_{X} \lesssim 20 \mathrm{TeV}\right)$, a conservative estimate of the flux can be made as follows. The interstellar gas which mostly consists of hydrogens has a local density $n_{\text {gas }} \simeq 0.8 \mathrm{~cm}^{-3}$ near the Sun. We assume that the gas moves with $v \simeq 30$ $\mathrm{km} / \mathrm{sec}$ relative to the Earth, comparable to the revolution speed of the Earth around the Sun. Then the flux will be estimated as

$$
\phi_{+} \simeq \frac{m_{H} n_{g a s}}{m_{X}} v f_{+}^{\prime}
$$

where $f_{+}^{\prime}$ denotes a fraction of the mass density of the CHAMPs in the interstellar gas. Similar to the previous case, it is natural to evaluate it as

$$
f_{+}^{\prime} \simeq \frac{1}{2} \frac{\Omega_{X} h^{2}}{\Omega_{b} h^{2}}
$$

Here $\Omega_{b}$ stands for the density parameter of the baryons and we will use $\Omega_{b} \simeq 0.05$ in our computation. The abundance of the positive CHAMPs in the sea water for $m_{X} \lesssim 20 \mathrm{TeV}$ is then calculated as

$$
\left(\frac{n_{X}}{n_{H}}\right)_{\text {Earth }} \simeq 6 \times 10^{-5}\left(\frac{\mathrm{GeV}}{m_{X}}\right) \Omega_{X} h^{2} .
$$


Our main result is given in Fig. 2 where the expected abundance of the positive CHAMPs inside the sea water is plotted as a function of their mass. The experimental bounds are also indicated in the same figure. We find that for $m_{X} \lesssim 1 \mathrm{TeV}$ our estimated abundance exceeds the experimental bounds even if we take the lowest allowed reheating temperature of $1 \mathrm{MeV}$. Thus any stable CHAMP weighing less than $1 \mathrm{TeV}$ should be excluded. On the contrary we find some allowed region for higher masses if the reheat temperature is low enough. For instance, the CHAMPs with $m_{X}=10^{4}, 10^{5} \mathrm{GeV}$ can survive the experimental constraints if $T_{R} \lesssim 1,300 \mathrm{GeV}$, respectively.

Before closing, we would like to mention some implications of our results to model building. In SUSY standard models, the LSP is stable under the R-parity conservation. Which particle becomes the LSP depends on the mechanisms of supersymmetry breaking and its mediation to the MSSM sector. In some scenarios, the stau, the superpartner of the tau lepton, which is charged becomes the LSP. Now our result excludes such a stable stau, as far as its mass is below $1 \mathrm{TeV}$ as is expected from the naturalness argument in the Higgs sector. Another implication is to gauge mediated supersymmetry breaking (GMSB). There the lightest messenger particle may be stable and charged. The mass of the particle is expected to be much larger than $1 \mathrm{TeV}$. Our consideration then allows existence of such a particle, which makes the model building of GMSB more flexible.

\section{ACKNOWLEDGMENTS}

We thank T. Moroi for delightful conversations. A.K. also thanks Sh. Matsumoto and F. Takayama for discussions and Y. Kiyo for assistance on numerical computation. This work was supported in part by the Grant-in-aid from the Ministry of Education, Culture, Sports,

Science and Technology, Japan, priority area (\#707) "Supersymmetry and unified theory of elementary particles," and in part by the Grants-in-aid No.11640246 and No.12047201. 


\section{REFERENCES}

[1] A. De Rújula, S.L. Glashow and U. Sarid, Nucl. Phys. B333 (1990) 173.

[2] S. Dimopoulos, D. Eichler, R. Esmailzadeh and G.D. Starkman, Phys. Rev. D41 (1990) 2388.

[3] For a review, see H.P. Nilles, Phys. Rep. 111 (1984) 1.

[4] S. Dimopoulos, G.F. Giudice and A. Pomarol, Phys. Lett. B389 (1996) 37.

[5] E.W. Kolb and M.S. Turner, The Early Universe (Addison-Wesley Publishing Company, 1990).

[6] P.F. Smith and J.R.J. Bennet, Nucl. Phys. B149 (1979) 525.

[7] P.F. Smith et al., Nucl. Phys. B206 (1982) 333.

[8] T.K. Hemmick et al., Phys. Rev. D41 (1990) 2074.

[9] P. Verkerk et al., Phys. Rev. Lett. 68 (1992) 1116.

[10] T. Yamagata, Y. Takamori and H. Utsunomiya, Phys. Rev. D47 (1993) 1231.

[11] G.F. Giudice, E.W. Kolb and A. Riotto, hep-ph/0005123.

[12] J. McDonald, Phys. Rev. D43 (1991) 1063.

[13] D.H. Lyth and E.D. Stewart, Phys. Rev. Lett. 75 (1995) 201; Phys. Rev. D53 (1996) 1784 .

[14] T. Asaka, K. Hamaguchi and K. Suzuki, Phys. Lett. B490 (2000) 136.

[15] D.E. Groom et al. (Particle Data Group), Eur. Phys. J. C15 (2000) 1.

[16] K. Griest and M. Kamionkowski, Phys. Rep. 333 (2000) 167. 


\section{TABLES}

\begin{tabular}{lll}
\hline \hline$\left(n_{X} / n_{H}\right)_{\text {Earth }}$ & mass & Reference \\
\hline$<4 \times 10^{-17}$ & $m_{X}=5-1600 m_{p}$ \\
$<6 \times 10^{-15}$ & $m_{X}=10^{5}-3 \times 10^{7} \mathrm{GeV}$ \\
$<7 \times 10^{-15}$ & $m_{X}=10^{4}, 6 \times 10^{7} \mathrm{GeV}$ \\
$<9 \times 10^{-15}$ & $m_{X}=10^{8} \mathrm{GeV}$ \\
$<3 \times 10^{-23}$ & $m_{X}=1000 m_{p}$ \\
$<2 \times 10^{-21}$ & $m_{X}=5000 m_{p}$ \\
$<3 \times 10^{-20}$ & $m_{X}=10000 m_{p}$ \\
$<1 \times 10^{-29}$ & $m_{X}=30-400 m_{p}$ \\
$<2 \times 10^{-28}$ & $m_{X}=12-1000 m_{p}$ \\
$<1 \times 10^{-14}$ & $m_{X}>1000 m_{p}$ \\
$<(0.2-1) \times 10^{-21}$ & $m_{X}=6-350 m_{p}$ & {$[0]$} \\
\hline \hline
\end{tabular}

TABLE I. Experimental bounds on the number density of CHAMPs with charge +1 relative to hydrogens inside sea water. $m_{p}$ represents the proton mass. 


\section{FIGURES}

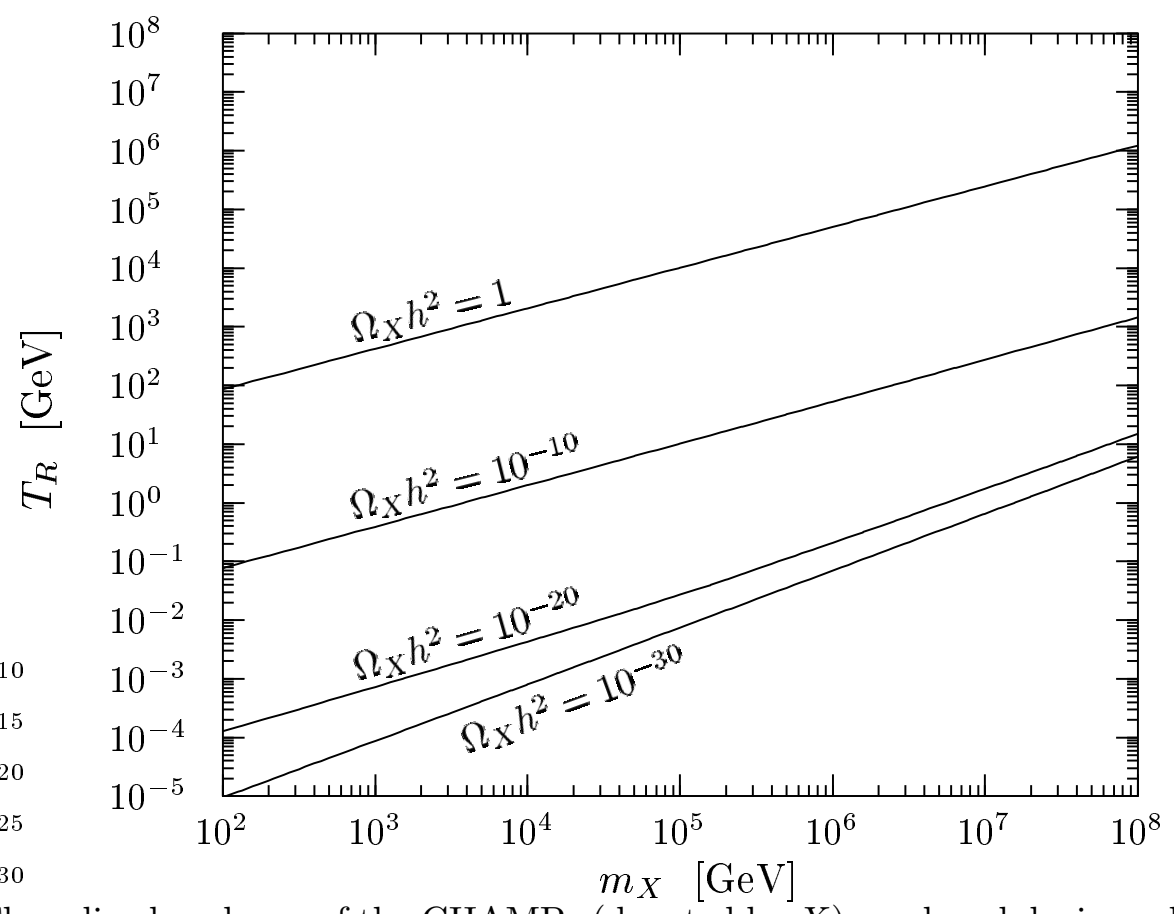

FIG. 1. The relic abundance of the CHAMPs (denoted by $X$ ) produced during reheating. Each line indicates a constant contour for $\Omega_{X} h^{2}=1,10^{-10}, 10^{-20}, 10^{-30}$ in the $m_{X}-T_{R}$ plane, where $m_{X}$ is the CHAMPs mass and $T_{R}$ is the reheating temperature of the Universe. In this calculation, we fixed $g_{*}(T)$ to $g_{*}=106.75$. For low reheat temperature, the relic abundance of the CHAMPs becomes drastically small. 


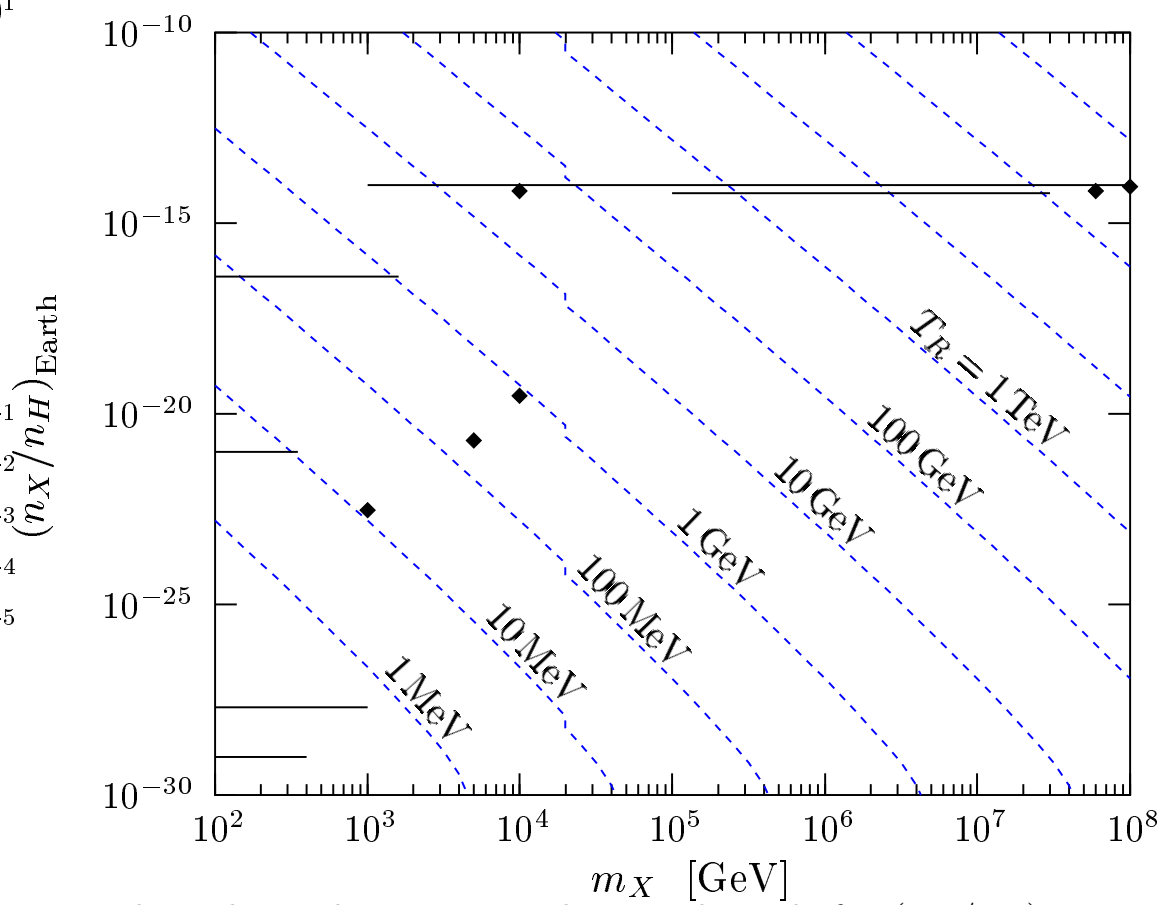

FIG. 2. Numerical results and experimental upper bounds for $\left(n_{X} / n_{H}\right)_{\text {Earth }}$ as a function of $m_{X}$. Broken lines indicate numerical results of our calculation for some different reheat temperatures $T_{R}=1 \mathrm{MeV}, 10 \mathrm{MeV}, \cdots$, where we used Eq. (14) for $m_{X} \geq 20 \mathrm{TeV}$ and Eq. (17) for $m_{X}<20 \mathrm{TeV}$. Solid lines and dots are experimental upper bounds for number density of the CHAMPs in the sea water [6-10], which are summarized in Table 1. 